\begin{tabular}{|l|l|c|c|}
\hline Eiszeitalter u. Gegenwart & $\mathbf{2 8}$ & $\begin{array}{c}195-199 \\
1 \mathrm{Abb} .\end{array}$ & Öhringen/Württ. 1978 \\
\hline
\end{tabular}

\title{
Ein frühpleistozänes Kalkartefakt von Würzburg-Schalksberg
}

\author{
AlfRed Rust *) \\ Excavations, limestone-implements, Homo beidelbergensis, animal bones
}

K u r z f a s s u n g : In den Jahren 1966 und 1976 wurden bei Ausschachtungsarbeiten in Würzburg-Schalksberg zahlreiche mauerzeitliche Faunenreste aus alten Ablagerungen des Mains geborgen. Zusammen mit den nichtumgelagerten, z. T. bearbeiteten Knochen wurden Werkzeuge aus Kalk entdeckt, die in altpleistozäner Technik hergestellt wurden.

\section{[An Old-Pleistocene Limestone-Implement at Würzburg-Schalksberg]}

Abstract: During excavations in the old sediments of the Main in 1966 and 1976 at Würzburg-Schalksberg the team found numerous bones according to the type of Mauer. Together with the bones which are not transported but worked on they found limestone-implements made in the old-pleistocene technic.

In den Jahren 1966 und 1976 stieß man bei Ausschachtungsarbeiten zur Errichtung von Universitätsgebäuden am Schalksberg in Würzburg auf reichgegliederte mauerzeitliche Fossilreste. Die Bergung der Tierrelikte unter Leitung von Prof. R. RutTe (1967) mußte im angeschnittenen Bereich der Vorkommen unter höchstem Zeitdruck kurzfristig durchgeführt werden.

Im Grabungssektor wurden zahlreiche Muschelkalkstücke freigelegt, von denen einige Exemplare als artefaktverdächtig von dem Geophysiker Dr. K. ERnstson geborgen wurden.

Zur Beurteilung liegen mir 5 Stücke aus der Grabung Schalksberg vor, von denen zwei durch Menschenhand geformt wurden. Als einleitenden Beitrag zur Gesamtpublikation scheint es mir sinnvoll, eines dieser Artefakte als zeitgebunden technisch charakterisierend zu beschreiben. Eine größere Anzahl von Kalkstücken ist nach der Präparierung der in erhärteten Sanden eingebackenen Faunenreste zu erwarten.

Das aus vielschichtigem Muschelkalk gefertigte Werkzeug, dem Cromersande anhaften, ist 10,4 cm lang, im Mittelteil 6,4 cm breit und im Schnitt 2,5 cm hoch. Die Oberseite (Abb. 1 a) ist, wie die Kanten, ganzflächig leicht windgeschliffen, die Unterseite (Abb. 1 c) nur im oberen Drittel entsprechend dem dunkel gefärbten Bereich, der experimentell bei Auflage auf einer Tischplatte etwas aufragt und vom Wind unterlaufen werden konnte. Ausprägungen dieser Art können in Nord- und Süddeutschland in unübersehbarer Fülle (auch an Kalkstücken) beobachtet werden. Der Windschliff bewirkte auf unserem Artefakt an Ober- und Unterseite kleinräumige Stufenbildungen, bedingt durch unterschiedliche Härte der dünnen Schichten (Rust 1971). Auf der Oberseite sind einige schwache, wohl durch metallische Bergungswerkzeuge verursachte Kratzer markiert.

Das aus der Fundschicht vom Schalksberg geborgene Artefakt ist, wie zu erwarten, in einer Technik zugerichtet worden, die für die Handhabungen des Frühmenschen kennzeichnend ist!

Wahrscheinlich wurde das Werkzeug nach dem Windschliff zum zweiten Male genutzt. Darauf deutet u. a. die zweite "typische“ Hohlbucht oben links (Abb. 1 a) hin, die gepunk-

*) Anschrift des Verfassers: Dr. A. R u s t, Am Rehm 52, 2070 Ahrensburg. 

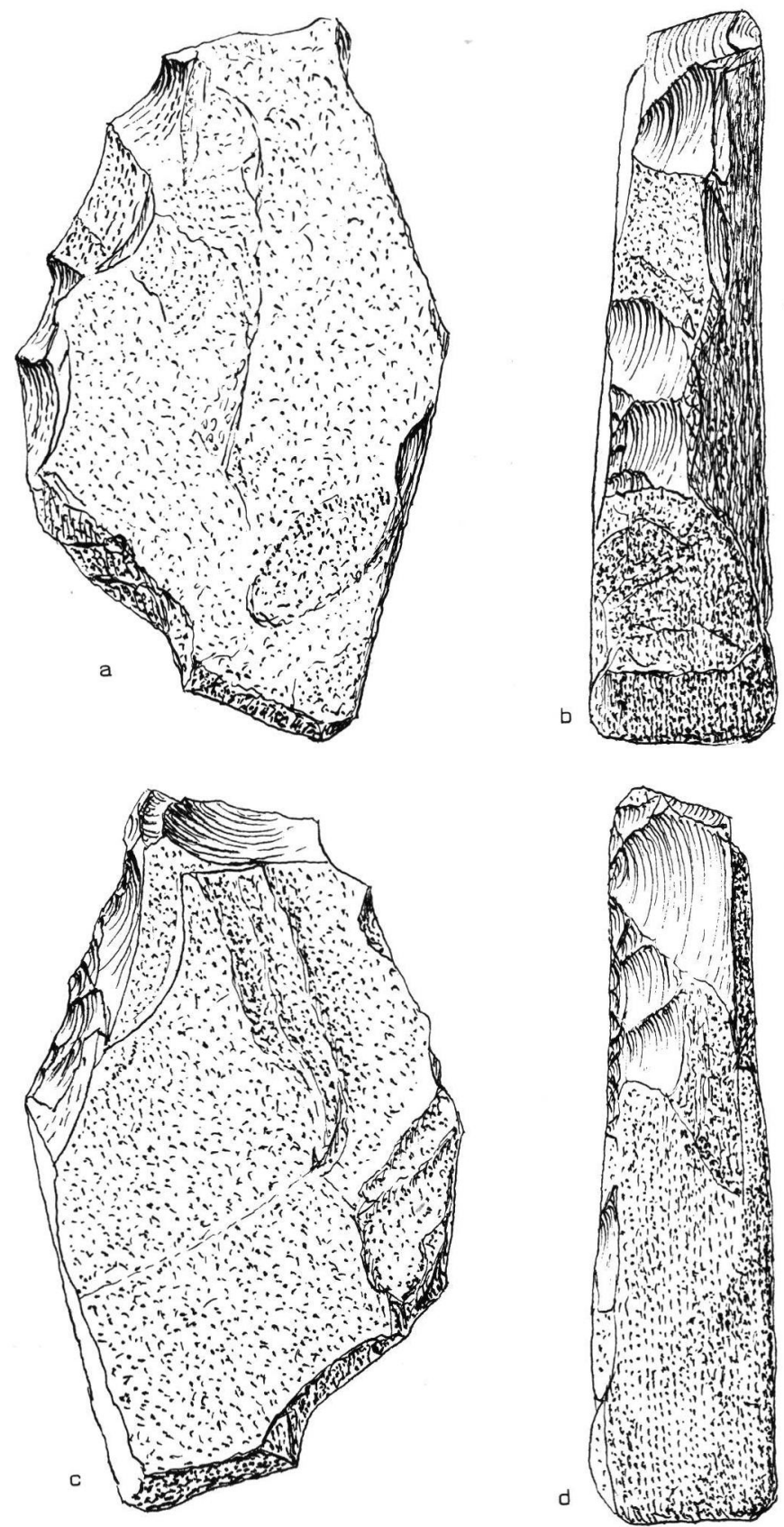

Abb. 1: Ein aus Muschelkalk in altpleistozäner Technik hergestellter Querschaber von Würzburg-Schalksberg, Gr. 2 : 3 . 
tet gezeichnet ist. Sie ist gleichartig windgeschliffen wie die ganze Oberfläche, die „frischen“ jüngeren, nichtgeschliffenen Abschlagpartien sind in den Zeichnungen durch Strichführung wiedergegeben.

Diese Zweitnutzung von Artefakten war in der Frühzeit ein viel geübtes Brauchtum! Dazu ein Kurzreferat: In jüngeren Entwicklungsperioden konnte man bei geringem Arbeitsaufwand in kurzer Zeit eine Vielzahl von Abschlägen, Klingen, Handspitzen und dgl. anfertigen. In der Frühzeit bedurfte es oft mehr als hundert Zuschlägen, um ein Artefakt, vielleicht durch "handliche Behinderungen“ bedingt (RUST 1965), im Griffbereich zum Schutze vor Verletzungen zuzuformen. Solche oft aus sehr hartem Material gefertigten, weggeworfenen, angewitterten oder vom Wind angeschliffenen Artefakte wurden von Nachfolgern aufgenommen und nur an der Schneidenpartie durch einen oder wenige Abschläge nachgeschärft, wieder verwendet.

Bei dem Artefakt vom Schalksberg handelt es sich um einen Querschaber oder Querhobel, einen Werkzeugtyp, der bis zu den Anfängen der Verwendung von geschlagenen Gesteinsstücken durch den Urmenschen zu beobachten ist.

In Abb. 1 b blicken wir auf die nach rechts aufgekantete Seitenfläche der Abb. 1 a. Am Oberende verdeutlicht das Abschlagnegativ einen von rechts geführten Schlag, darunter liegt die Markierung eines von links angesetzten Schlages. Die quergestellte Kante zwischen diesen Abschlagbahnen war ursprünglich messerscharf und verdeutlicht jenen angestrebten Abschnitt, der bei der Bearbeitung von Knochen oder Hölzern wirksam angewandt wurde. Alle weiteren Abschlagbahnen, auch in Abb. $1 \mathrm{c}$ und d, markieren vermutlich die Bearbeitung zur Entschärfung der Griffpartien, was z. T. nicht ganz gesichert ist.

Wir sprachen für die Querschaber von einem technisch uralt gebundenen Regelverhalten des Frühmenschen. Eine der Stützen zur Verdeutlichung dieser Auffassung ist die Technik, in der die als älteste Werkzeuge des Menschen aufgefaßten „Pebble tools“ zugerichtet wurden. Das Ausgangsmaterial zur Anfertigung dieses oder „des Uraltartefaktes“ der Menschheit (was nicht zu stimmen braucht) ist ein rundliches, etwas flach gedrücktes, handliches Geröllstück, das im bearbeiteten Zustand bei der Suche heute am auffälligsten ist. Trennt man von diesem Rohling an einer Kante eng nebeneinander, alternierend mittels Schlagstein zwei Abschläge ab, so erzeugt man einen Querschaber mit ideal scharfer Arbeitskante. Bei gleichartiger Fortsetzung der Kantenbearbeitung entsteht ein MehrfachQuerschaber oder Wellenschaber. Der Querschaber ist neben dem Nasenschaber ein prägendes Kennzeichen der Entwicklung des Frühmenschen auf technischer Ebene, wie wir sie u. a. auch an den Artefakten des Heidelbergers von Mauer und an den aus Süßwasserflint gefertigten "Eolithen“ aus dem Cantalgebiet in reicher Fülle eindeutig ausgeprägt beobachten können. Solche „Primitiv-Typen“ wurden im gesamten Pleistozän verwendet, z. B. vorrangig auch im Clactonien.

Eine technisch basierte Spezialuntersuchung könnte vielleicht Anhalte bieten, um wahrscheinlich zu machen, daß der Frühmensch zur Herstellung seiner rundlichen primitiven Waffen wie Speeren, Lanzen, Keulen, Wurfhölzern und dgl. bei noch ungelenker Hand seine Werkzeuge vorwiegend hobelartig schob oder zog mit ausgestreckter Hand, die wie auch der Unterarm bei hohem Krafteinsatz noch nicht „allgemein“ zum Körper hin eingewinkelt wurde. Ähnliche „Bindungen“ scheinen auch für die Nasenschaber maßgeblich gewesen zu sein (Rust 1965).

Als Anhang: Die Kalkartefakte vom Schalksberg wurden von einem Geophysiker geborgen. Im Gebiet der Alb leistete der Physiker und Ministerialrat Dipl.-Ing. Alfred ForstMeyer (1974) eine Pionierarbeit von hoher zukunftswirksamer Bedeutung - u. a. neben Windschliffstraten mit dem Nachweis zahlreicher Besiedlungsvorkommen mit industriell „typischen“ Artefakten aus Kalk! 
Von den Prähistorikern werden Kalkwerkzeuge in der Regel abgelehnt, in Bindung an eine veraltete Lehrmeinung. Diese Ablehnung kann nicht auf eine negativ verlaufene praktische Erprobung zurückgehen, d. h. auf die Herstellung und Anwendung von Werkzeugen aus Hartkalken. Praktisch negativ verlaufene Untersuchungsergebnisse auf dieser Ebene sind auch nie veröffentlicht worden.

Wesentliche Erkenntnisverluste erwachsen uns durch diese Ablehnung in Form einer Abbremsung der förderlichen Hilfseinsätze von Amateuren, die sehr wesentliche Beiträge für den Fortschritt geleistet haben. Als sehr betrüblich muß man z. T. den kürzlich von ADAM (1976) veröffentlichten Aufsatz auffassen, in dem - bezogen auf den Fundraum des Schädels von Steinheim - alle Kalkwerkzeuge als natürliche Erscheinungsformen unbegründet (!) abgelehnt werden und aufgefordert wird, sie als Schutt zu betrachten und nicht zu sammeln. Es ist natürlich recht interessant, von klimagebundenen, örtlichen tierischen Erscheinungsformen zu hören, aber solche gibt es z. B. in ganz Europa. Einmalig und nicht nur als Begleiterscheinung ist auf der Erde hingegen der Schädel von Steinheim und für die Anthropologie von außerordentlicher Bedeutung. Von hohem Wert ist dazu für die Prähistorie die Erlangung der Kenntnis, wie wir die Steinheimer Menschen in den Ablauf des technischen Fortschritts einzufügen haben, und zwar auch in Richtung auf Homo sapiens sapiens.

An die Amateure, vor allem auch aus dem Raume Steinheim, ist die Bitte zu richten, alle artefaktverdächtigen Kalkstücke zu sammeln und mit Schichtangaben aufzubewahren. Soweit ersichtlich führt nur dieser Weg zur „industriellen Belebung“ des Menschentyps von Steinheim, die zu kennen international von hoher Bedeutung ist. Unfaßbar ist auch die „Ächtung“ des „Faustkeiles“ von Steinheim als Fälschung des verstorbenen, mir persönlich bekannten, achtbaren Schulrates J. Itermann. Dieser „Faustkeil“ ist kein typischer Faustkeil, sondern ein in drei technischen Spezialmethoden gefertigtes faustkeilartiges, rechtshändiges Unikum, passend zu dem Schädel als anthropologisches Unikum. Das BifaceArtefakt von Steinheim ist ein Pendant zu den Biface-Werkzeugen von Swanscombe (mit dem Kalottenteil); außerdem liegen beide Vorkommen nicht auf primärer Lagerstätte, sondern sie sind umgelagert worden.

Über der „warmzeitlichen Schädelschicht" wurden in Steinheim Sedimente angereichert und in verstärkter Mächtigkeit auch in der dann folgenden „Kaltzeit“. Diese Auffüllung des Flußbettes bewirkte einen beträchtlichen Anstieg des Flußwasserspiegels, so daß in der Folge ehemals trockene Uferpartien im Oberlauf verfrachtet wurden. Es gibt keine Begründung, auszuschließen, daß (nach vielen gleichartigen Beispielen) auch Artefakte des Steinheimers verfrachtet wurden, die heute in den „Kaltschichten“ eingebettet sind. Unter den aus den „Kaltzeitschichten“, und zwar aus der Profilwand entnommenen Artefakten liegen auch verlagerte Werkzeuge vor, die aus quarzitischem Sandstein gefertigt wurden, und zwar in einer Technik, wie sie für den Homo beidelbergensis von Mauer charakteristisch ist.

Selbstverständlich kann man mit einem Kalkwerkzeug z. B. einen nach dem Lehringer Vorbild gespeerten Elefanten aufbrechen, aus der Decke schlagen, zerwirken und auch dessen Knochen bearbeiten. Dem Verfasser des oben genannten Aufsatzes im Kosmos ist zu empfehlen, ein von ihm „anerkanntes“ Silexartefakt formgerecht aus Muschelkalk nachzuformen und mit diesem im Beisein von Studenten und Amateuren z. B. ein Rind oder ein sonstiges Tier aufzubrechen, den Erfolgsablauf zu publizieren und zumindest unserem Nachwuchs damit eine Basis zu ebnen zur Erkenntnis, daß der Frühmensch sein Leben nicht nach unseren $z$. T. ausgefallenen, unfundierten und verkrampften Theorien gestaltete, sondern in seinem jeweiligen Jagdrevier das beste ihm zugängliche Rohmaterial zur Werkzeugherstellung nutzte. 
Beispielhaft zum Thema ist auch die Verwendung von Muschelkalk am Schalksberg. Nach Auswertung durch E. RuTTE ist zu erwarten, daß nach den bisher vorliegenden Veröffentlichungen der Nachweis erbracht wird, daß die Anreicherung und die Vielfalt der Tierknochen zumindest zum Teil auch auf ein „Regelverhalten“ des mitwirkenden Menschen zurückzuführen ist - des Schalksberger Heidelbergers, der am Ort besseres Rohmaterial vorfand als sein Vetter bei Mauer mit dem quarzitischen Sandstein, dazu der Ostafrikaner mit löcherigem Basalt oder gar der Peking-Mensch mit Quarz, der den geächteten Muschelkalk wohl als Himmelsgabe angesehen hätte.

$$
\text { Schriftenverzeichnis }
$$

Adam, K. D. (1976): Steinheim an der Murr - eine Fossilfundstelle von Weltrang. - Kosmos, 76, 10: 411-419, 15 Abb.; Stuttgart.

Forstmeyer, A. (1974): Die südliche Frankenalb, Refugium des Menschen im gesamten Pleistozän. - Naturhist. Ges. Nürnberg.

Rust, A. (1965): Uber Waffen- und Werkzeugtechnik des Altmenschen. - 68 S., 25 Abb., 5 Taf.; Neumünster (Wachholtz Verl.).

- (1971): Werkzeuge des Frühmenschen in Europa. - 72 S., 29 Abb.; Neumünster (Wachholtz Verl.).

Rutre, E. (1967): Die Cromer - Wirbeltierfundstelle Würzburg-Schalksberg. - Abh. naturw. Ver. Würzburg, 8: $1-26,8 \mathrm{Abb}$.; Würzburg.

- (1976): Würzburg-Schalksberg. Eine Fossilfundstätte der Superlative. - Information, 10: 26-30; Würzburg. 